\title{
A Low Leakage Input Dependent ONOFIC Approach for CMOS Logic Circuits
}

\author{
Vijay Kumar Sharma ${ }^{1}$, and Manisha Pattanaik ${ }^{2}$
}

\begin{abstract}
With the rise of the number of electronic portable devices, the importance of leakage power reduction techniques are increasing. Leakage power dissipation has become one of the critical issues in modern integrated circuit. This work focuses on the low power circuit design, which is showing an ever increasing growth with the scaling of technology nodes. Supply voltage and threshold voltage of the device are scaled down to maintain the performance at lower technology nodes. Reduction in threshold voltage causes leakage power in the concerned device. The number of gates per chip has been increasing with technology scaling which resulted in large leakage power dissipation. The demand of low power integrated circuits is increasing to improve the performance of portable systems. In this research paper, a low leakage circuit level approach is proposed in order to overcome the leakage power dissipation. 4:1 multiplexer is designed and simulated at $32 \mathrm{~nm}$ technology node by using proposed approach and the results are compared with the conventional 4:1 multiplexer circuit.
\end{abstract}

Keywords-Device scaling, leakage power, nanoscale CMOS, VLSI circuits.

\section{INTRODUCTION}

$\mathrm{T}$ HE leakage power dissipation factor plays an important role because it degrades the performance of various parameter of a system at lower technology nodes, hence the factor cannot be neglected. The parameter includes packaging, reliability, cost, etc of the systems. While the leakage power dissipation always existed in the past but they had little impact. It is a dominant part of total power dissipation below $100 \mathrm{~nm}$ technology nodes. The leakage power dissipation has its origin from a number of factors which include increase in transistor count, operating frequency and most important is the technology scaling [1, $2]$.

The scaling of the technology is done as per the ITRS (international technology roadmap for semiconductor) recommendation. The linear dimension are scaled by a factor of 0.7 often termed as scaling parameter and the area is reduced by 2 units as per Moore's law [3, 4]. The technology scaling means reduction in different parameters of the

Vijay Kumar Sharma ${ }^{1}$ is with the Department of Electronics \& Communication Engineering, Shri Mata Vaishno Devi University, Katra, 182320 India.

Manisha Pattanaik ${ }^{2}$ is with the Department of information technology, $\mathrm{ABV}$ - Indian Institute of Information Technology \& Management, Gwalior, 474015 India. devices. The power consumption exceeds the expected values in integrated circuits thereby, need for the techniques and besides mechanisms for heat removal. Together with heat removal mechanism, a number of techniques had been employed which include work at process level, architecture level and at circuit level. Transistor delay times decreases by more than $30 \%$ per technology generation, resulting in doubling of microprocessor performance every two years [5, 6]. Supply voltage has been scaled down in order to keep the power consumption under control. Hence, the transistor threshold voltage has to be commensurately scaled to maintain a high drive current and achieve performance improvement. In comments on this problem at the 2002 International Electron Devices Meeting, Intel chairman Andrew Grove cited off-state current leakage in particular as a limiting factor in future microprocessor integration. It is to be remembered that all the parameters are not scaled beyond a particular limit, it may result in short channel effect and many more. For digital applications, the most undesirable short channel effect is the reduced gate threshold voltage at which the device turns on. The goal of this research paper is to propose a leakage power reduction approach at circuit level design to minimize the off-state leakage.

The organization of the paper is as followed. Section II is related to background of this field. Proposed technique is presented in section III. Results and outcomes are discussed in section IV. Finally this research work is concluded in section $\mathrm{V}$.

\section{BACKGROUND}

There are number of leakage reduction techniques available in literature survey. They have own advantages and disadvantages. The important techniques are presented in this section. Multi-threshold CMOS (MTCMOS) techniques introduces insertion of high threshold device in series to low threshold devices. In this technique, the sleep control scheme is used for efficient power management [7]. Low threshold means high leakage current but contrary high operating speed whereas high threshold indicates low leakage power. However using two types of transistors, the circuit becomes complex as well as costlier. The drawbacks include; area and delay affects, not suitable for sequential circuit, reduce leakage power in standby mode.

A low threshold device enhances speed while a high 
threshold device reduces the leakage power. A higher threshold and lower threshold voltage is assigned to the devices depending on critical and noncritical paths respectively. For noncritical path a higher threshold voltage is introduced in order to reduce the leakage current. In order to maintain the performance, a low threshold transistor is used irrespective of high leakage. Therefore no additional leakage transistor is used to reduce leakage and thus both low power and high performance is achieved simultaneously [8]. Threshold assignments require special kinds of algorithms.

When more than one transistor in a stack is turned off, the sub threshold leakage current flowing through a stack of series connected transistor reduces. The effect is known as transistor stacking. The leakage of a two transistor stack is about an order of magnitude less than the leakage in a single transistor. Mechanism like input dependence increases the number of off transistor in a stack of transistor by properly choosing the input of gates and thereby reduces the standby leakage current [9].

Input pattern of each gate affects the sub threshold as well as gate leakage current. It is to be remembered that the leakage of a transistor in a stack is a function of number of transistor and input pattern [10]. In control point insertion technique, the selection of gates is done in a proper manner so that the gates that contribute greater leakage are selected first. The approach too comes with a drawback of the area and delay penalty. It includes gate level strategy as well as circuit level strategy. Based on the selective insertion of control point, leakage reduces to $70 \%$ of the total leakage with minimum increase in delays and area [11]. Process variations are the important parameters in nanoscaled regime and calculated in circuit level leakage minimization technique INDEP [12].

\section{INPUT DEPENDENT ONOFIC APPROACH}

The schematic arrangement of the proposed leakage reduction approach is shown in fig. 1 It uses the concepts of ONOFIC approach [6] as well as INDEP approach [12]. ONOFIC block operates as per the logic available at output terminal. The logic circuit inside the pull-up network affects the operation of MP2 transistor. It will be turned-on when logic at node N2 is high and turned-off when logic at node $\mathrm{N} 2$ is low. The input voltage $\mathrm{V}_{0}$ manages the logic path between pull-up network and output node. Insertion of MP2 transistor between the pull-up network and output node makes a transistor stack and reduces large leakage power.

Table 1 explain the operation of input dependent ONOFIC CMOS inverter logic for reducing the leakage current. For input logic low, both PMOS and MP2 will be turned-on to provide the conducting path between power supply and output node. PMOS and NMOS are the transistors in pull-up and pull-down network respectively. For input logic high, MN1 and MP1 will be turned-on and provide the conducting path between output node to ground.

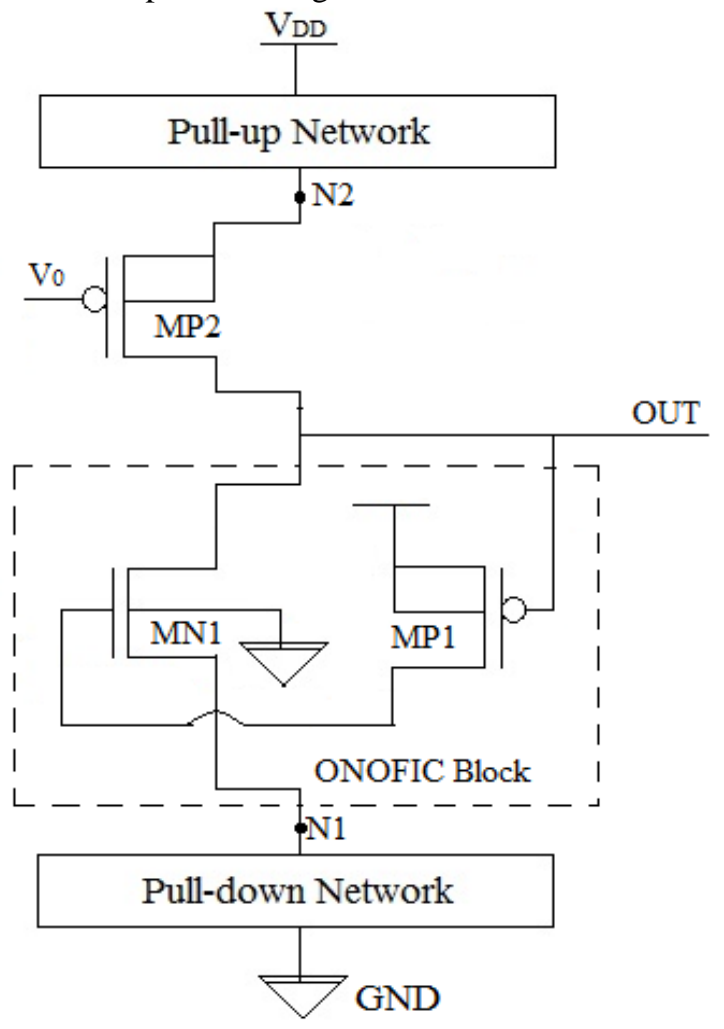

Fig. 1 Schematic arrangement of input dependent ONOFIC approach

Proposed approach uses the same threshold voltage devices within the logic circuit. Therefore, it is easy to develop the efficient leakage reduction technique without using any bias controller. This idea overcomes the problem of handing different threshold voltages controller circuits.

TABLE I

OPERATING STATUS OF THE TRANSISTORS IN INPUT DEPENDENT ONOFIC CMOS INVERTER

\begin{tabular}{lccccc}
\hline \hline Input Level & PMOS & MP1 & MP2 & MN1 & NMOS \\
\hline Logic Low & On & Off & On & Off & Off \\
Logic High & Off & On & Off & On & On \\
\hline \hline
\end{tabular}

The primary function of the logic circuits is provided by DC characteristic and transient response and is judged before any type of performance analysis. Fig. 2 shows the comparative DC characteristics of CMOS inverter circuit using input dependent ONOFIC approach. Electrical simulations are carried out for $\mathrm{VDD}=0.8 \mathrm{~V}$ at room temperature at $32 \mathrm{~nm}$ BSIM4 CMOS PTM technology node. Transient responses of CMOS inverters are given in fig. 3 with same parameter assumptions where signal $\mathrm{v}($ in) shows the common input to all circuits. Fig. 3 depicts that output of input dependent ONOFIC and conventional CMOS circuits have the same behavior. 


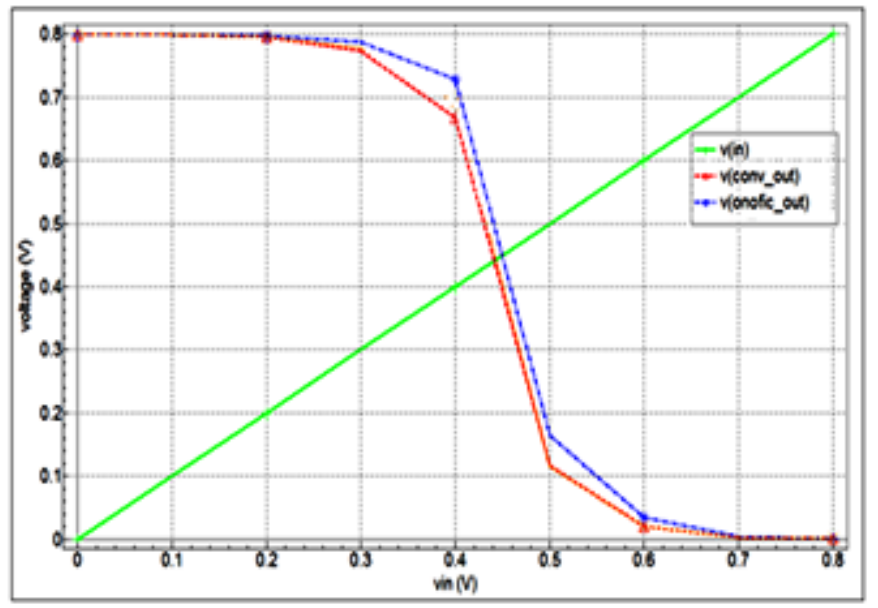

Fig. 2 DC characteristics for CMOS inverter

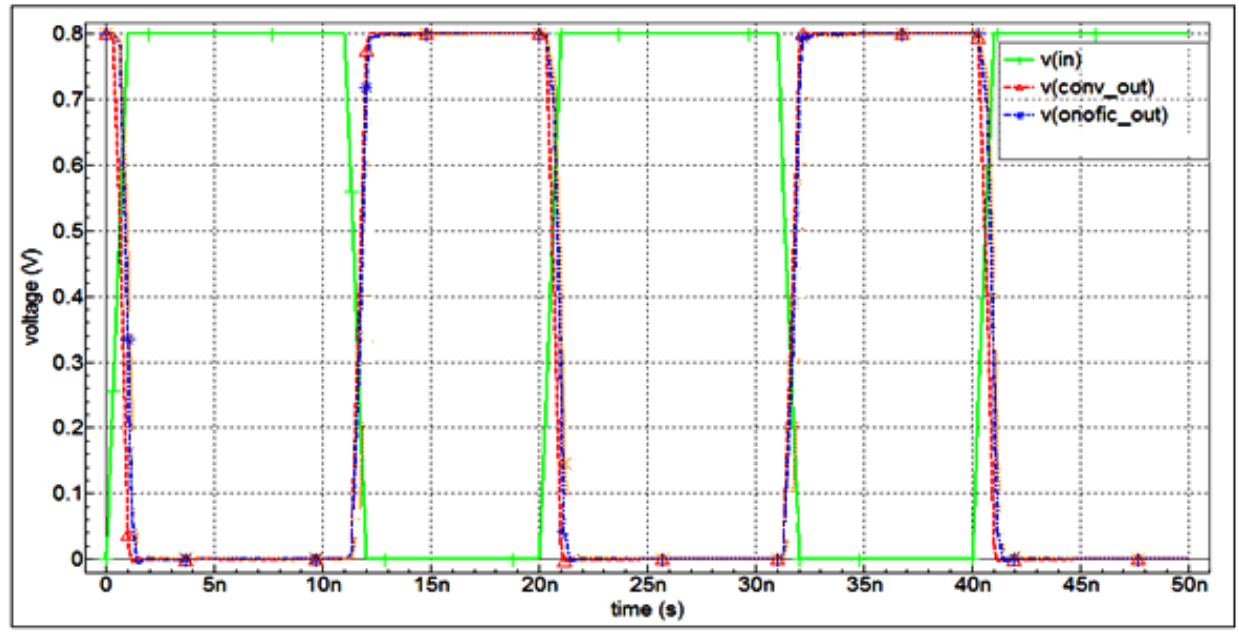

Fig. 3 Transient responses of input dependent ONOFIC and conventional CMOS inverter

\section{RESUlTS AND DISCUSSIONS}

This section covers the simulation study of different leakage reduction techniques. CMOS 4:1 multiplexer circuit is simulated for comparison purpose. All experimental data were obtained at $32 \mathrm{~nm}$ BSIM4 bulk CMOS PTM technology node. Channel width of NMOS transistor was $2 \mathrm{X}$ of the respective NMOS channel length and for PMOS; it was sized to $3 \mathrm{X}$ of the respective pull-down NMOS size. Electrical simulations were carried out through HSPICE tool. HSPICE generates different SPICE model files for different sets of parameters. These results are taken at room temperature with some parameter assumptions. For making a fair comparison among the techniques, these parameter assumptions are equal to all. Numbers of basic logic gates are required to design the 4:1 multiplexer as shown in fig. 4. NAND, OR and inverter circuits are designed by using CMOS logic.

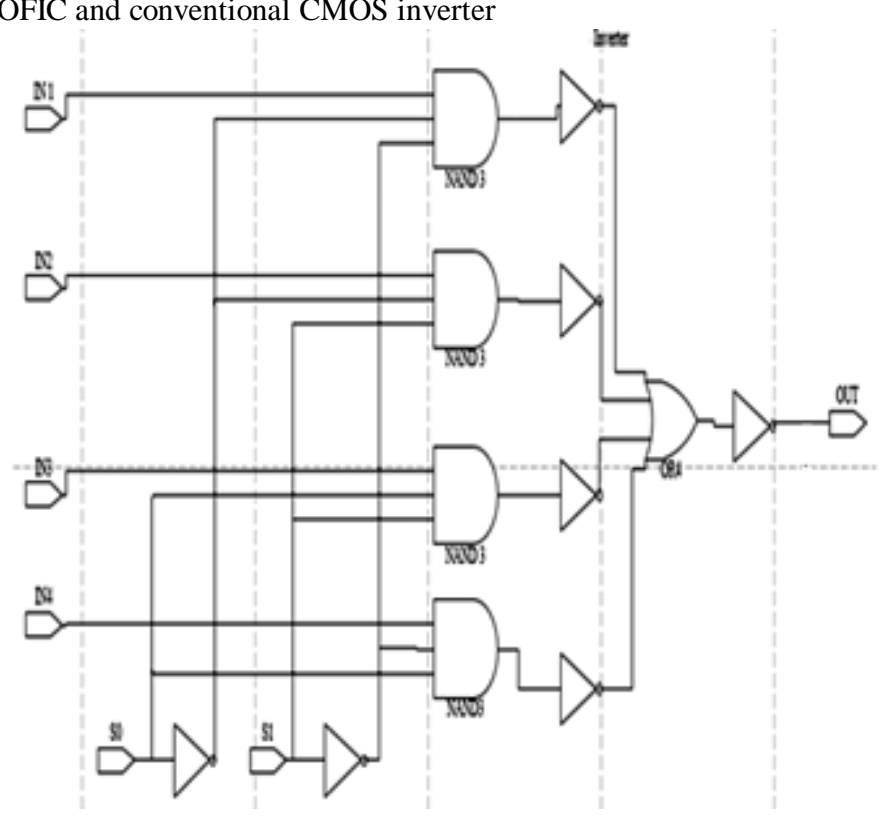

Fig. 4 Gate level diagram of 4:1 multiplexer

The proposed low leakage input dependent ONOFIC approach is applied for designing 4:1 multiplexer circuit. All the basic logic gates are designed by using proposed 
approach and then replaced the conventional gates of fig. 4 by the proposed gates to get the benefit of low leakage circuit. The output characteristic of the circuit in terms of transient behaviour of the proposed approach is shown in fig. 5 for power supply $1 \mathrm{~V}$. The primary function of the circuit is verified in fig. 5.

Since the voltage reduction provides an effective means for leakage power reduction. Since we are reducing the supply voltage, different results have been taken at different voltages. Leakage power and delay of the circuits have the trade-offs and these are plotted by varying supply voltage VDD from $0.4 \mathrm{~V}$ to $1 \mathrm{~V}$ and depicted in fig. 6.

The sizing of the extra inserted transistors (MN1, MP1 and MP2) is the important concern of the proposed approach. Efficient and proper sizing of the transistors gives the better result in terms of leakage power and propagation delay. Fig. 7 shows the sizing effect for an input dependent ONOFIC 4:1 multiplexer circuit. Multiply number indicates that the widths of MN1, MP1 and MP2 transistors are the times of pull-up/pull-down NMOS and PMOS transistors in the logic circuit. It can be observed from the fig. 7 that if the widths of extra inserted transistors are increasing then the leakage power dissipation is also increasing because the leakage current is directly depending on the feature size of the transistors. The benefits of increased sizing of the transistors are the reduction in the propagation delay and hence enhance the speed of the logic functions.

\section{V.CONCLUSION}

Today's era is the small portable devices era like smart phones, tablets/laptops, personal digital assistant, Wi-Fi devices and other electronic gadgets. These portable devices require long life battery with higher performance of the applications which are fabricated in a small chip area to optimize the space capability of the devices. Leakage power is focusable fact of each new technology node of the applications in nanoscale regime. In this research article; circuit level leakage minimization approach for CMOS logic circuits is proposed. 4:1 multiplexer circuit is simulated with the help of HSPICE tool and compared the results on the parameters of leakage power and propagation delay. From the simulation results, it is concluded that proposed approach provides the most efficient leakage minimization.

\section{REFERENCES}

[1] V. K. Sharma and M. Pattanaik, "VLSI scaling methods and low power CMOS buffer circuit", Journal of Semiconductors, vol. 34, pp. 095001, 2013. https://doi.org/10.1088/1674-4926/34/9/095001

[2] B. Nikolic, "Design in the power-limited scaling regime", IEEE Transactions on Electron Devices, vol. 55, no. 1, pp. 71-83, 2008. https://doi.org/10.1109/TED.2007.911350

[3] G. E. Moore, "Cramming more components onto integrated circuits," Proceedings of The IEEE, vol. 86, no. 1, pp. 82-85, 1998. https://doi.org/10.1109/JPROC.1998.658762
[4] K. J. Kuhn, "Moore's Law past 32nm: Future Challenges in Device Scaling", Proceedings of 13th International Workshop on Computational Electronics, pp.1-6, 2009. https://doi.org/10.1109/iwce.2009.5091124

[5] B. C. Paul, A. Agarwal and K. Roy, "Low-power design techniques for scaled technologies", Integration, the VLSI journal, vol. 39, no.2, pp. 6489, 2006.

[6] V. K. Sharma, M. Pattanaik and B. Raj, "ONOFIC approach: Low power high speed nanoscale VLSI circuits design", International Journal of Electronics, vol. 101, pp. 61-73, 2013. https://doi.org/10.1080/00207217.2013.769186

[7] K. Roy, S. Mukhopadhyay and H. Mahmoodi-Meimand, "Leakage current mechanisms and leakage reduction techniques in deepsubmicrometer CMOS circuits", Proceedings of the IEEE, vol. 91, no. 2, pp. 305-327, 2003. https://doi.org/10.1109/JPROC.2002.808156

[8] Y.-T. Ho and T.-T. Hwang, "Low power design using dual threshold voltage," in IEEE ASP design automation conference, pp.205-208, 2004.

[9] V. K. Sharma and M. Pattanaik, "Techniques for LowLeakage Nanoscale VLSI Circuits: A Comparative Study," Journal of Circuits, Systems, and Computers, vol. 23, no. 5, pp. 1450061(1-21), 2014.

[10] Y. ho, C. Chang, and C. Su, "Design of a sub-threshold supply bootstrapped CMOS inverter based on an active leakage current reduction technique," IEEE transaction on circuit and system express brief -II, vol. 59, no.1, pp- 55-59, 2012.

[11] H. Rahman and C. Chakrabarti, "An Efficient Control Point Insertion Technique for Leakage Reduction of Scaled CMOS Circuits", IEEE Transactions on Circuits and Systems-II: Express Briefs, vol. 52, no. 8, pp. 496-500, 2005

[12] V. K. Sharma, M. Pattanaik and B. Raj, "PVT variations aware low leakage INDEP approach for nanoscale CMOS circuits", Microelectronics Reliability, vol. 54, pp. 90-99, 2014.

https://doi.org/10.1016/j.microrel.2013.09.018 


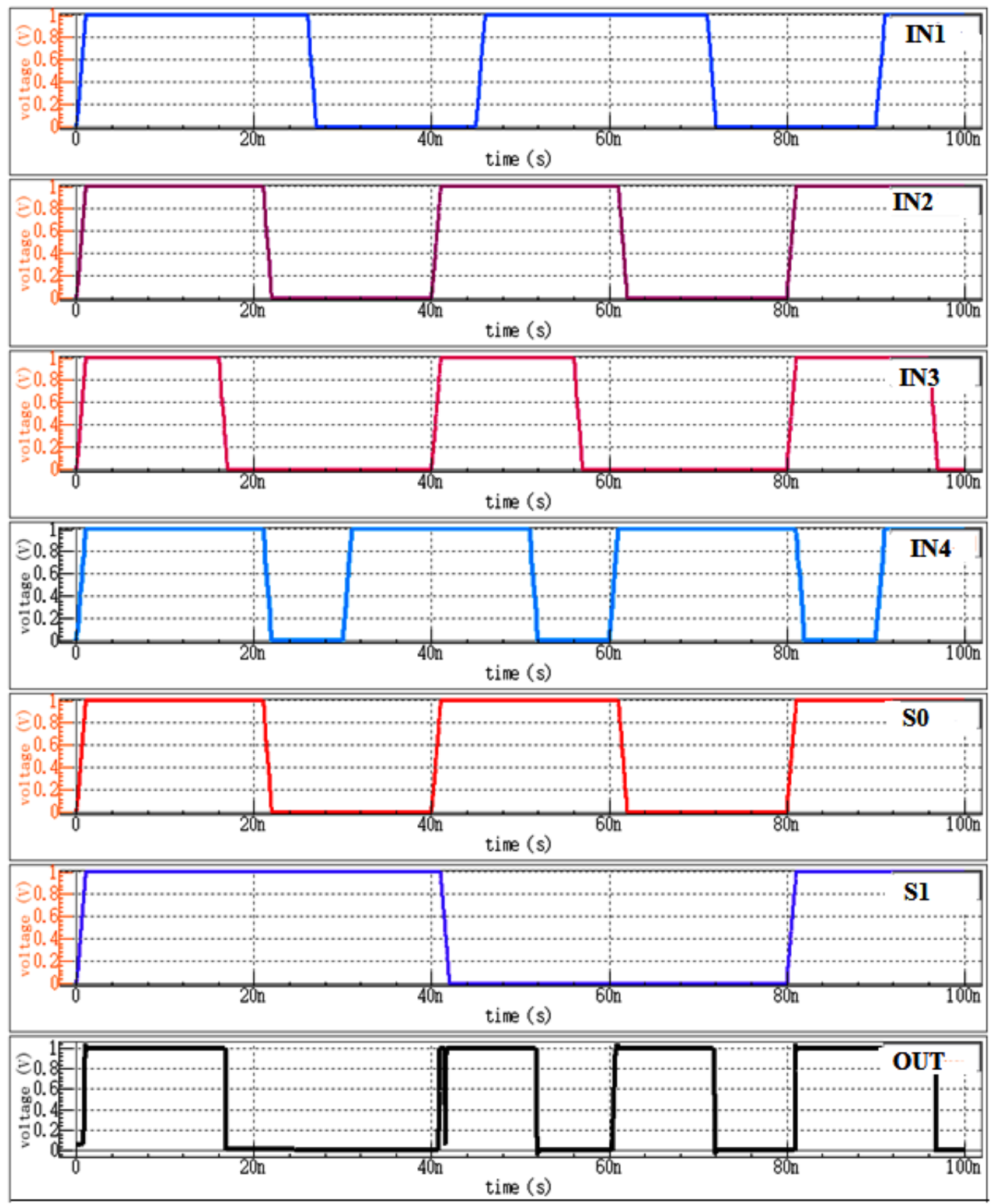

Fig. 5 Simulated output waveform of CMOS 4:1 multiplexer 


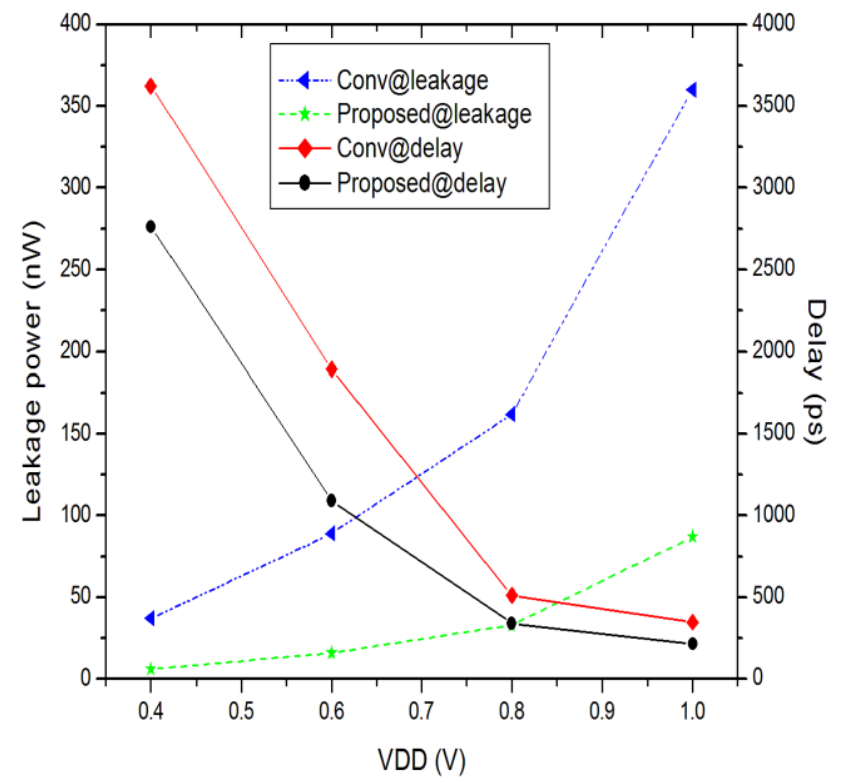

Fig. 6 Leakage power and delay vs VDD for 4:1 multiplexer

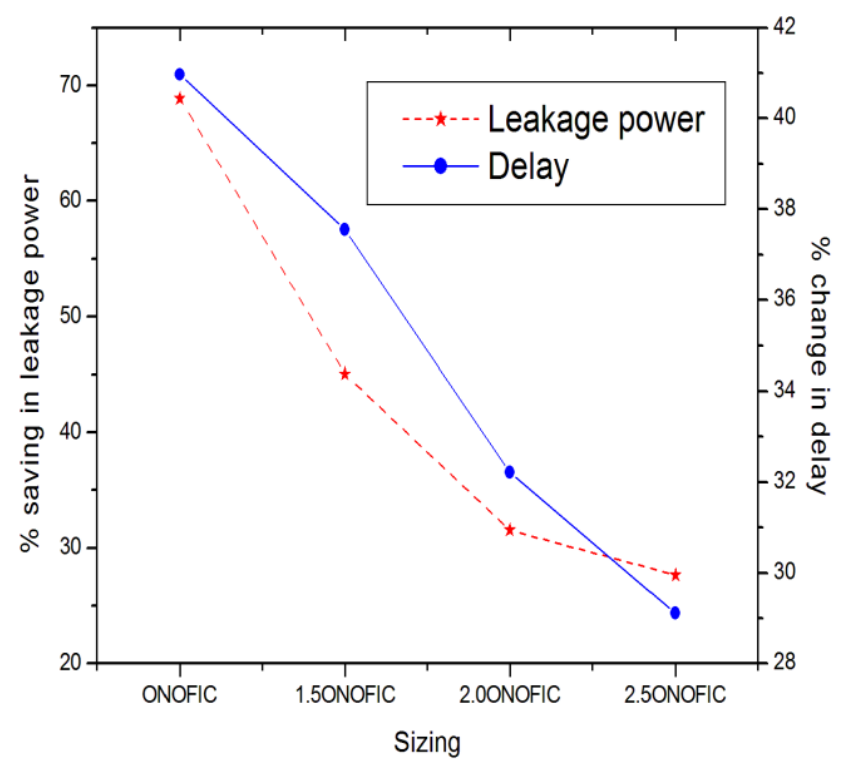

Fig. 7 Sizing effect of input dependent ONOFIC approach for 4:1 multiplexer 